IRRITABLE BOWEL SYNDROME

\title{
Sex specific alterations in autonomic function among patients with irritable bowel syndrome
}

\author{
K Tillisch, E A Mayer, J S Labus, J Stains, L Chang, B D Naliboff
}

Gut 2005;54:1396-1401. doi: 10.1136/gut.2004.058685

Background: Irritable bowel syndrome (IBS) is associated with increased psychological symptoms, early life stressors, and alterations in visceral perception and brain responses to noxious visceral stimuli. The autonomic nervous system (ANS) is a likely mediator for these brain-gut interactions. The few studies directly examining ANS measures have been suggestive of alterations in some IBS patients, but no studies

See end of article for authors' affiliations

.....................

Correspondence to: Dr K Tillisch, CNS/WH: Center for Neurovisceral Sciences and Women's Health, CURE Building 115, Room 223, 11301 Wilshire Boulevard, Los Angeles, CA 90073, USA; ktillisch@mednet.ucla.edu

Revised version received 6 May 2005

Accepted for publication 9 May 2005

Published online first

28 May 2005 to date have examined the potentially critical variables of sex differences or response to visceral stimulation.

Aims: (1) To test differences in ANS function during rest and during a visceral stressor (rectosigmoid balloon distension) between IBS patients and healthy control subjects. (2) To examine the role of sex on the autonomic responses of IBS patients.

Methods: Baseline autonomic measures were evaluated from 130 Rome I positive IBS patients and 55 healthy control subjects. Data were also collected from a subset of 46 IBS patients and 16 healthy control subjects during a sigmoid balloon distension study. Heart rate variability measures of peak power ratio (PPR) and peak power high frequency (PPHF) were analysed to assess sympathetic balance and parasympathetic response, respectively. Peripheral sympathetic response was measured by skin conductance.

Results: IBS patients showed a greater skin conductance response to visceral distension than controls. IBS patients had higher PPR and lower PPHF across conditions. Male IBS patients had higher skin conductance and PPR than females and lower PPHF.

Conclusions: IBS patients have altered autonomic responsiveness to a visceral stressor, with increased sympathetic and decreased parasympathetic activity. These differences are predominantly seen in males. in ritable bowel syndrome (IBS) is a functional gastrointestinal disorder affecting up to $15 \%$ of the US population, predominantly females. ${ }^{1}$ It is characterised by abdominal pain or discomfort combined with alterations in bowel function. Despite major advances in the conceptualisation of IBS pathophysiology as a disorder of altered brain-gut interactions, ${ }^{2}$ there is currently no general agreement on the relative roles of visceral perception and autonomic nervous system (ANS) dysfunction in the generation or maintenance of IBS symptoms. As early as 1928, it was hypothesised that dysfunction of the ANS contributed to the symptoms seen in IBS. ${ }^{3}$ The sympathetic and parasympathetic branches of the ANS mediate central nervous system (CNS) communication with the gut, largely through modulation of the third ANS branch, the enteric nervous system. Through its three divisions, the ANS modulates and coordinates gastrointestinal motility, secretion, and immune function. ${ }^{45}$ Modulation of the ANS, including increased sympathetic nervous system (SNS) and decreased parasympathetic nervous system (PNS) activity, is an important part of the organism's response to stress. The resulting changes in gastrointestinal function may provide the mechanism linking life stress and affective states to IBS symptoms.

Heart rate variability (HRV) and skin conductance (SC) can be used to measure ANS function. The high frequency component of HRV predominantly reflects cardiovagal activity, the ratio of low to high frequency reflects SNS balance, and SC reflects the SNS mediated response of the eccrine sweat glands. ${ }^{67}$ While these measures of ANS activity do not directly describe ANS regulation of gastrointestinal function, Buysschaert et al have shown a close association between cardiac and gastric autonomic function. ${ }^{8}$ Additionally, Emmanuel and Kamm have shown that cardiovagal tone, as measured by HRV, correlates with rectal mucosal blood flow and colonic transit, further supporting the relationship between cardioautonomic measures and gastrointestinal function. ${ }^{9}$

Previous studies investigating ANS function in IBS patients have shown inconsistent results, possibly due to differences in sample characteristics, assessment parameters, and conditions under which measurements were taken. ${ }^{10-20}$ IBS is thought to be a stress responsive disorder, and the ANS output provides an easily measurable physiological response to stress. As might be expected, increased SNS activity and decreased PNS activity are the most frequently noted differences when IBS patients are compared with healthy controls. ${ }^{12} 15162122$ Studies of short term ANS activity in IBS patients have examined subjects at rest or during postural change, while other studies have examined longer term recordings throughout the day or during sleep. It is plausible that important differences between IBS and control subjects may be more prominent during a stressor.

Although recent evidence has confirmed that sex differences exist among IBS patients in terms of perception, ${ }^{23}$ central response to visceral stimuli, ${ }^{24}$ and colonic transit response to pharmacological treatment, ${ }^{25}$ sex differences in the autonomic response have not been reported. Sex differences in ANS responses have been reported for healthy subjects, showing greater SNS responsiveness in males and greater PNS responsiveness in females. ${ }^{26}{ }^{27}$ Previous ANS

Abbreviations: IBS, irritable bowel syndrome; IBS-C, constipation predominant IBS; IBS-D, diarrhoea predominant IBS; IBS-A, alternating bowel habit of IBS; ANS, autonomic nervous system; PPHF, peak power high frequency; PPR, peak power ratio; SC, skin conductance; SNS, sympathetic nervous system; PNS, parasympathetic nervous system; $H R V$, heart rate variability; HAD, hospital anxiety and depression scale 
studies in IBS have either included only women or have not systematically examined sex, a potential explanation for the contradictory data that exist. Differences in ANS responses to specific stimuli may also play a role in producing sex based variations in IBS symptom patterns and in differential responses to some pharmacological agents. ${ }^{28} 29$

Psychological state may alter autonomic responses and has not always been well controlled for in previous IBS studies. Reduced HRV, likely mediated by increased sympathetic/ vagal balance, has been described in stress, depression, and panic disorders. ${ }^{30}$ One study in females has shown lower parasympathetic tone among IBS patients with comorbid anxiety/depression, ${ }^{10}$ indicating that psychological state must be addressed when assessing autonomic parameters.

In the current study, our aim was to test differences in ANS function during rest and with an acute visceral stressor (balloon distension) between IBS patients and healthy control subjects in the absence of concomitant depression or anxiety. Because of previously noted sex differences in IBS prevalence, response to visceral stimulation, and medication effectiveness, we also wanted to examine the role of subject sex on these responses. We hypothesised that: (1) IBS patients will have increased resting SNS and decreased resting PNS activity compared with control subjects; (2) IBS patients will have increased SNS and decreased PNS responsiveness to a relevant visceral stimulus compared with healthy control subjects; and (3) male IBS patients will have a greater sympathetic/vagal balance compared with female IBS patients. Based on these hypotheses we examined autonomic function at baseline and autonomic responses to rectosigmoid balloon distension in male and female IBS patients and healthy controls. No specific predictions were made for bowel habit differences in ANS response but these were examined in secondary analyses.

\section{METHODS}

\section{Patient population}

Baseline autonomic measures were evaluated from a total of 130 IBS patients and 55 healthy control subjects. A subset of 46 patients with IBS (as defined by the Rome I criteria) ${ }^{31}$ and 16 healthy control subjects also participated in a sigmoid balloon distension study. Control or IBS subjects who had participated in a balloon distension study within the previous six months were excluded. No correlations were seen between ANS measures and whether subjects had a history of participation in an a prior barostat study. Subjects were recruited by advertisement and from the Functional Bowel Disorders Clinic at UCLA. All subjects were screened for an affective disorder using the hospital anxiety and depression scale $(\mathrm{HAD})^{32}{ }^{33}$ or SCL-90. ${ }^{34}$ Subjects with HAD scores $>11$ or SCL-90 scores for global severity index $>63$ were excluded from the study. Bowel habit predominance was defined by responses to the Rome supportive symptoms question list. ${ }^{31}$ Patients showing symptoms of both diarrhoea predominant IBS (IBS-D) and constipation predominant IBS (IBS-C) were classified alternating bowel habits (IBS-A). Symptom severity was measured on a scale of 1 to 4 . Patients also rated the intensity and unpleasantness of their overall IBS symptoms over the past six months and the past 24 hours on a $20 \mathrm{~cm}$ visual analogue scale with anchoring word descriptors. ${ }^{35}$ All study protocols were performed after approval by the West Los Angeles Veterans Administration Institutional Review Board. Informed consent was obtained from all subjects.

\section{Rectosigmoid distension protocol}

Patients underwent a standard barostat protocol, as previously described. ${ }^{36}$ A double balloon catheter was placed using sigmoidoscopic guidance with the proximal tip $40 \mathrm{~cm}$ from the anal verge. One latex balloon was close to the tip (sigmoid) and the second placed with the distal attachment $4 \mathrm{~cm}$ from the anal verge (rectum). The catheter was attached to a computer operated distension device (barostat) which allowed for rapid phasic distensions of each balloon. After balloon placement there was a 20 minute rest period prior to beginning the balloon inflations. A rectal stimulus tracking protocol was used to determine a reliable discomfort threshold, as previously described ${ }^{37}$ Briefly, this consisted of a series of 14, 30 second inflations which were increased or decreased depending on the subject's judgment of the previous inflation as either causing discomfort or not. The discomfort threshold was determined as the average of the last six tracking inflations. The sigmoid stimulation protocol consisted of a series of 10, 30 second distensions at $60 \mathrm{~mm} \mathrm{Hg}$ over 10 minutes.

\section{ANS measurements}

Autonomic measurements were recorded for 10 minutes at the end of the 20 minute initial rest period in all subjects. In a subset of subjects, measurements were also taken during the last 10 minutes of a 20 minute rest period following sigmoidoscopic balloon placement, and during the 10 minute period of phasic sigmoid distensions. A two lead ECG and SC were continuously sampled at $500 \mathrm{~Hz}$ during these periods using a Biopac recording system and software (BioPac Inc., Santa Barbara, California, USA). A custom designed peak detection algorithm was used to determine the interbeat intervals for assessment of both average HR and as raw data for the analysis of HRV. SC was recorded from the first and second fingers of the left hand using a constant current method and averaged over the entire period. SC levels were transformed using a $\log ^{10}$ to correct for a positive skew. HRV measures included spectral analysis measures of high frequency peak power (PPHF) and the peak power ratio of low to high frequency (PPR) using the procedure of Fallen and colleagues. ${ }^{38}$ PPHF assessed vagal tone and PPR was used to assess the sympathetic/parasympathetic balance.

\section{Data analysis}

Analysis of variance (ANOVA) comparing male and female, IBS and control subjects, was used to examine baseline differences in ANS responses. Post hoc pairwise comparisons for the four separate groups were performed using the Bonferroni procedure. A mixed model approach was used to examine group differences in responsiveness to the visceral stressor. Compared with conventional repeated measures analyses, the mixed modelling approach provides greater statistical power, flexibility, parsimony, handles time varying covariates and missing data more effectively, and is less prone to false positives than ANOVA and MANOVA. ${ }^{39}$ Therefore, a mixed modelling approach for repeated measures specifying the best fitting covariance structure ${ }^{40}{ }^{41}$ was applied using a 2 group (IBS versus control) $\times 3$ condition (baseline, post-balloon placement, and sigmoid inflation) ANOVA design. For all of the analyses, unstructured covariance was used (SPSS 12.0; SPSS Inc., Chicago, Illinois, USA). Due to the restricted sample size in the healthy controls group, the influence of sex was examined only for IBS patients using a similar mixed model approach. Group differences in demographics, symptoms, and discomfort thresholds were assessed using $t$ tests for continuous variables and $\chi^{2}$ tests for categorical variables. Significance for all tests was set as $\mathrm{p}<0.05$.

\section{RESULTS \\ Subjects with baseline data \\ Characteristics}

Mean ages of the IBS patients and control subjects were 41.1 (10) years and 40.2 (11) years, respectively. The control 
Table 1 Baseline autonomic nervous system (ANS) measures

\begin{tabular}{|c|c|c|c|c|c|}
\hline \multirow[b]{2}{*}{ ANS measure } & \multicolumn{2}{|l|}{ Controls } & \multicolumn{2}{|l|}{ IBS } & \multirow[b]{2}{*}{$\begin{array}{l}\text { Subject group } \\
\text { effect }\end{array}$} \\
\hline & $\begin{array}{l}\text { Males } \\
(n=29)\end{array}$ & $\begin{array}{l}\text { Females } \\
(n=22)\end{array}$ & $\begin{array}{l}\text { Males } \\
(n=64)\end{array}$ & $\begin{array}{l}\text { Females } \\
(n=63)\end{array}$ & \\
\hline PPHF & $41.6(22.2)$ & 44.1 (18.9) & $31.5(13.8)$ & $45.2(20.8)$ & $p=0.001$ \\
\hline PPR & $2.0(1.5)$ & $1.6(0.9)$ & $3.2(2.6)$ & $1.7(1.1)$ & $p<0.001$ \\
\hline
\end{tabular}

group had $56 \%$ males $(n=31)$; the IBS group had 50\% males $(n=65)$. There were no significant age or sex differences between the IBS and control groups. IBS patients included $22 \%$ IBS-C (39\% male), $43 \%$ IBS-D (54\% male), and 35\% IBSA (52\% male). Autonomic data could not be analysed for four control subjects (two males, two females) and three IBS patients (one male, two females) secondary to collection artefact.

Baseline autonomic measures in IBS patients versus controls: group and sex comparisons

An overall significant effect of subject group was seen in baseline PPHF $(p=0.001)$. IBS males had the lowest values, with a significant difference between IBS males and both IBS females $(p=0.001)$ and control females $(p=0.044)$. A significant group effect was also seen in PPR $(p<0.001)$, with IBS males showing a significantly higher sympathetic ratio compared with IBS females $(p<0.001)$, control females $(p=0.003)$, and control males $(p=0.026)$. Mean values for PPHF and PPR are shown in table 1. No effects for sex or presence of IBS were found in baseline skin conductance.

\section{Bowel habits}

There was no significant difference in percentage of specific bowel habits between male and female patients with IBS. No significant association between bowel habit and baseline ANS measures was found (table 2).

\section{Subjects undergoing balloon distension protocol} Characteristics

IBS patients had a mean age of 40.8 years (range 24-68; SD 10.6). The group included 17 females and 29 males. Evaluation of bowel habit predominance showed: $39.1 \%$ IBS-D, $17.4 \%$ IBS-C, and $43.5 \%$ IBS-A. The control group included six males and 10 females and had a mean age of 38.0 years (range $21-56$; SD 11.5 ). There was no significant age difference between the groups. The majority of patients

Table 2 Baseline autonomic nervous system (ANS) measures of irritable bowel syndrome (IBS) patients by bowel habit

\begin{tabular}{llcr}
\hline \multirow{2}{*}{ ANS measure } & $\begin{array}{l}\text { IBS-A } \\
(\mathbf{n}=\mathbf{4 5})\end{array}$ & $\begin{array}{l}\text { IBS-C } \\
(\mathbf{n}=\mathbf{2 7})\end{array}$ & $\begin{array}{l}\text { IBS-D } \\
(\mathbf{n}=\mathbf{5 5})\end{array}$ \\
\hline SC & $4.3(4.0)$ & $3.0(1.4)$ & $3.9(3.6)$ \\
PPHF & $37.3(17.6)$ & $44.0(19.8)$ & $35.9(19)$ \\
PPR & $2.3(1.5)$ & $1.9(1.0)$ & $2.9(2.8)$ \\
\hline
\end{tabular}

Values are mean (SD)

IBS-C, constipation predominant IBS; IBS-D, diarrhoea predominant IBS; IBS-A, alternating bowel habit of IBS; SC, skin conductance; PPHF, peak power high frequency; PPR, peak power ratio.

Mean values for autonomic measures by bowel habit (IBS-C, IBS-D, IBSA) are shown. There was no significant difference between bowel habit subgroups for SC $(p=0.32), \operatorname{PPHF}(p=0.18)$, or PPR $(p=0.12)$.

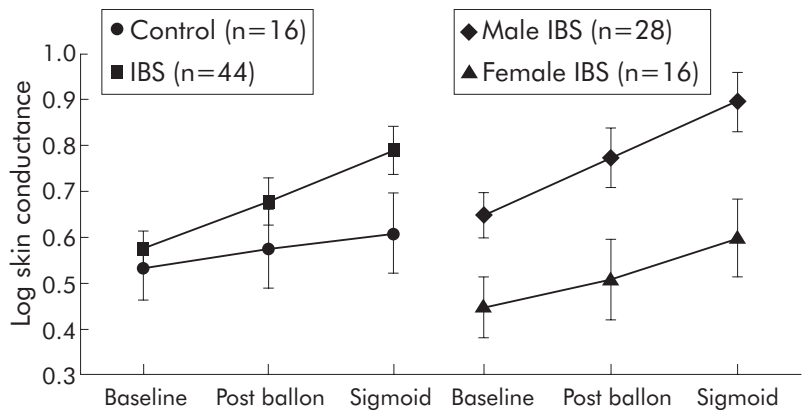

Figure 1 Skin conductance (SC) scores (with standard error bars) are shown at baseline, after balloon placement, and during sigmoid distension for control and irritable bowel syndrome (IBS) subjects, and for male and female IBS subjects. A significant condition $\times$ group interaction $(p=0.039)$ was seen, with higher SC in the IBS group versus the control group. A significant sex main effect $(p=0.010)$ was seen between male and female IBS patients, with higher SC in the male group.

(76\%) reported their overall IBS symptoms as moderate to severe (mild $7.9 \%$, moderate $42.1 \%$, severe $34.2 \%$, very severe $15.8 \%)$. No significant difference in self reported severity was observed between males and females. There were also no significant sex differences in the ratings of six month or 24 hour IBS symptom unpleasantness or intensity. Both male and female IBS patients had significantly higher mean scores for intensity and unpleasantness for the six month period than for the 24 hour period $(\mathrm{p}<0.001)$. The group of subjects with baseline data only was compared with the subgroup for whom balloon distension data were obtained, showing no differences in age, sex, symptom severity, or baseline values for SC, PPHF, or PPR. SC and HRV data were available for all 16 control subjects but SC data for two IBS patients and HRV data for six IBS patients could not be analysed secondary to collection artefact.

\section{ANS response to visceral stressor in IBS versus control subjects}

\section{SNS measures}

SC increased after balloon placement and again during sigmoid stimulation in both groups, and this change was greater in the IBS group (within subjects condition effect, $\mathrm{p}<0.001$; condition $\times$ group interaction, $\mathrm{p}=0.0390$ ) (fig $\mathrm{l}$ ). A trend towards higher SC in IBS subjects was seen during sigmoid inflation compared with controls (SC log 0.789 (0.37) $v 0.601(0.30) ; \mathrm{p}=0.06)$. IBS patients had overall higher PPR (group main effect, $\mathrm{p}=0.0068$ ) (fig 2).

\section{PNS measures}

There was a group main effect with lower PPHF in IBS subjects at all time points $(\mathrm{p}=0.044)$ (fig 3$)$. 


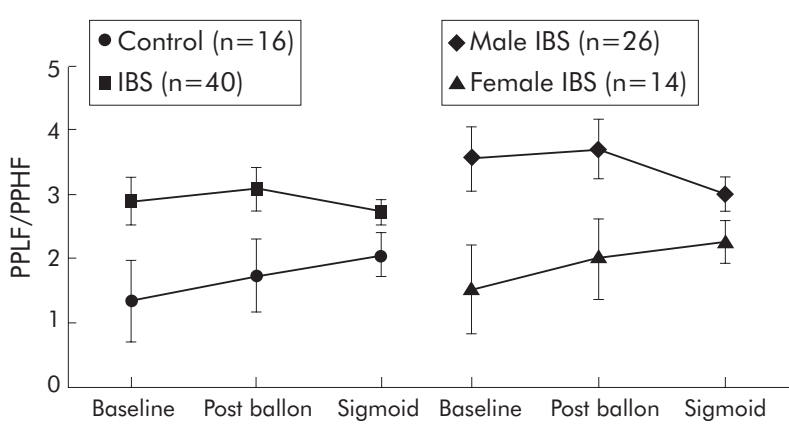

Figure 2 Peak power ratio of low to high frequency (PPLF/ PPHF) scores (with standard error bars) are shown at baseline, after balloon placement, and during sigmoid distension for control and irritable bowel syndrome (IBS) subjects, and for male and female IBS subjects. A group main effect $(p=0.0068)$ was seen for the IBS versus the control group, with higher sympathetic balance in the IBS group. A sex main effect $(p=0.0031)$ was seen between male and female IBS patients, with higher sympathetic balance in the male group.

\section{ANS response to visceral stressor in IBS males versus IBS females}

SNS measures

SC showed both a significant sex main effect $(p=0.010)$ and a significant condition interaction $(\mathrm{p}<0.001)$. As shown in fig 1 , this was due to a greater increase in SC for male IBS subjects over the course of the experiment. PPR was also higher in males across conditions $(\mathrm{p}=0.0031)$ (fig 2$)$.

\section{PNS measures}

A significant sex interaction $(\mathrm{p}<0.008)$ in PPHF appears to be due to greater parasympathetic tone in female patients at baseline, which subsequently declined with balloon placement and sigmoid distension (fig 3 ).

\section{Bowel habits}

No significant effect of bowel habit was seen on any of the ANS measures.

\section{Perception of visceral stimuli: tracking rectal} discomfort threshold

Mean discomfort threshold was significantly lower in IBS patients compared with healthy controls (35.5 (15) mm Hg $v$ 44.9 (11) $\mathrm{mm} \mathrm{Hg} ; \mathrm{p}=0.039$ ). There were no significant correlations between the autonomic parameters and tracking discomfort threshold.

\section{DISCUSSION}

The current study, in a sample of IBS patients without affective comorbidity and healthy control subjects, demonstrated evidence of higher SNS activity and lower cardiovagal activity in the patient group. Within the IBS population there were significant sex differences, with greater SNS activity and lower PNS activity in males compared with females. Taken together, these findings suggest that differences in ANS function measures between IBS patients and controls may be primarily due to greater sympathetic/vagal balance in the male IBS population.

\section{Autonomic response in IBS versus control subjects}

The results indicate greater sympathetic responsiveness in IBS patients compared with controls, as measured by PPR. IBS patients also showed lower parasympathetic activity compared with controls, as measured by PPHF. This shift towards greater sympathetic and lower parasympathetic balance is consistent with a recent study evaluating isometric handgrip, orthostatic testing, and expiratory:inspiratory ratio

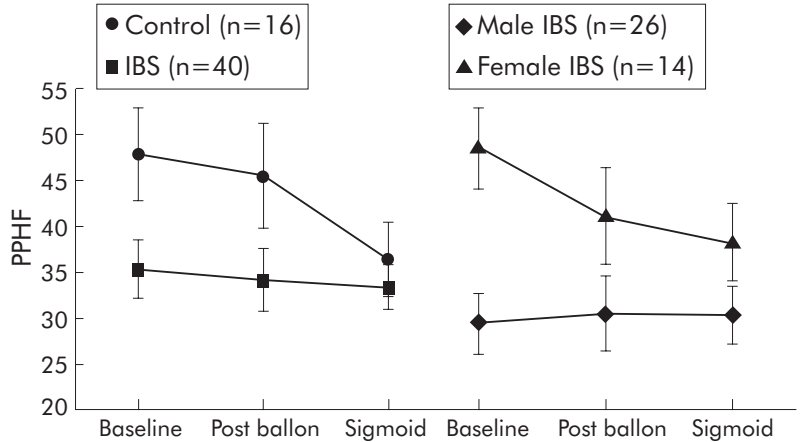

Figure 3 Peak power high frequency (PPHF) scores (with standard error bars) are shown at baseline, after balloon placement, and during sigmoid distension for control and irritable bowel syndrome (IBS) subjects, and for male and female IBS subjects. A group main effect $(p=0.044)$ was seen with lower parasympathetic tone in the IBS group compared with controls. A sex main effect $(p=0.008)$ was seen between male and female IBS patients, with lower parasympathetic tone in the male group.

in IBS patients. ${ }^{21}$ Recent hypotheses have suggested that parasympathetic vagal responses serve as a "brake" to control sympathetic responses to stress. The pattern of greater sympathetic responses with less parasympathetic activity in IBS males may therefore indicate a deficient parasympathetic "braking" of the autonomic response to distension stress in IBS patients. ${ }^{42}$ Greater stress responsiveness with increased SNS activity may play a role in IBS symptom pathogenesis. Immune modulation occurs via neural mechanisms, thus a generalised shift in ANS function towards higher sympathovagal balance may lead to a relative proinflammatory state, as may be seen in post-infectious IBS. The stress response in animal models is associated with increased distal colonic motility and decreased upper gut motility. Similarly, alterations in gastrointestinal motility may occur in IBS patients with increased sympathetic balance. Previously, patients with IBS alone were also found to have greater sympathetic/vagal balance during sleep compared with controls but IBS patients with dyspepsia did not. ${ }^{12}$ Subgrouping by upper gastrointestinal symptoms was not tested in this study.

\section{Autonomic response in males versus females}

The autonomic differences seen in IBS appear to be primarily due to changes in male subjects. IBS males had lower parasympathetic tone and showed no parasympathetic responsiveness over varying experimental conditions compared with female IBS patients. They also had greater sympathetic responsiveness than their female counterparts, as measured by SC and PPR. When compared with healthy male controls, male IBS patients had significantly higher sympathovagal balance whereas no differences were noted between female controls and IBS females.

This dichotomy by sex is an important finding and may be one of the factors involved in differential responses to drug therapy seen in male and female IBS patients. It may also explain some of the inconsistencies in previous reports of autonomic function in IBS. Two studies have shown impaired vagal function in a group of IBS patients that was predominantly male, ${ }^{19} 20$ findings that were not seen in a female sample of IBS patients. ${ }^{15}$ Increasingly, sex differences have been noted in other physiological responses of IBS patients. Notably, rectal distension thresholds have been shown to be lower in females compared with males. ${ }^{23} 43$ Sexual dichotomy has been observed in cortico-limbic interactions also. Specifically, a recent brain imaging study has shown that males with IBS compared with females have more dominant activation of the dorsal pons, periaqueductal 
gray, and insula during visceral stimulation. ${ }^{24}$ The insula is thought to be involved in regulation of visceromotor activity, particularly sympathetic responses, ${ }^{44}$ and the periaqueductal gray integrates autonomic and antinociceptive stress responses ${ }^{45}$ These findings are consistent with our current data, showing greater sympathetic responses in males with IBS. Additionally, previously reported higher rectal distension thresholds in male IBS patients ${ }^{23}$ support the hypothesis of a more effective antinociceptive response to visceral stimuli. In response to visceral stimuli, females may be less likely to mobilise an antinociceptive response and in a rectal distension protocol have been shown to activate brain areas associated with hypervigilance (dorsal anterior cingulate cortex) and affective response to the visceral stimulus (ventromedial prefrontal cortex). ${ }^{24}$ These cumulative findings suggest that males have autonomic dysregulation as a key feature of IBS whereas females may have greater alterations in visceral hypersensitivity.

\section{Bowel habit differences}

No differences in ANS responses were found for the three bowel habit groups. Data from previous studies has been inconsistent regarding bowel habit effects, with complicating factors being difficulties in classification and possible multiple physiological pathways for a similar symptom picture (for example, slow and normal transit constipation). SNS balance has been described as greater in IBS-D by one group ${ }^{22}$ and greater in IBS-C by another group. ${ }^{15}$ Elsenbruch and Orr found greater PPR and lower PPHF in females with IBS-D in response to a meal compared with both controls and IBS-C. ${ }^{17}$ Comparisons between studies is difficult due to vast differences in experimental conditions. Varying definitions, or inclusion of IBS-A patients in either IBS-D or IBS-C subgroups, may also effect ANS outcomes. Additionally, fluctuation between bowel habit is common, particularly in the poorly characterised IBS-A group. ${ }^{46}$ Our data do not support an interaction between bowel habit and autonomic parameters, nor did a recent study by Waring et al, although neither study was specifically designed to study this interaction. $^{21}$

\section{Symptom severity}

Different patterns of ANS activity in female patients with severe versus non-severe abdominal pain have been described. In one study, severe but not moderate or mild pain was associated with lower vagal tone. ${ }^{16}$ In another, IBS-C patients showed lower vagal tone than IBS-D, but only in the subgroup with severe pain. ${ }^{15}$ We did not find a relationship between symptom severity and our ANS measures. Methodological differences including acute versus 24 hour ANS measures, symptom assessment scale, and population differences may account for the variation in findings. It is relevant to note that the symptom reports in our sample for the last 24 hours were substantially less than those for chronic symptoms (which may reflect a global impression of symptom impact rather than a true average symptom level). Future studies of the role of ANS activity in symptom generation should examine both measures of acute symptom severity as well as subjective evaluations of chronic disease severity.

\section{Conclusions}

Decreased parasympathetic tone and increased sympathetic activity with altered ANS responsiveness to a visceral stimulus are prominent in IBS male patients, but not IBS females. This may be due to sex differences in cortico-limbic processing of visceral stimuli. These findings emphasise the need to take sex into account when studying physiological parameters in IBS and may explain some of the discrepancies seen in previous studies. Further studies should include the use of multiple forms of visceral, somatic, and psychological stressors to examine situation specific responses. Studies that also include measures of CNS activity (such as brain imaging or brain evoked potentials) and neuroendocrine variables will be especially useful in evaluating the role of autonomic response in IBS patients. Further research into the autonomic response with attention to sex based differences will be important in further elucidating the pathophysiology and potential treatments of IBS.

\section{ACKNOWLEDGEMENTS}

Supported in part by NIH grants NR007768 (BN) and P50 DK64539 (EM), and VA Medical Research (BN). The authors would like to thank Teresa Olivas for data management and editorial assistance in the preparation of the manuscript.

\section{Authors' affiliations}

K Tillisch, CNS/WH: Center for Neurovisceral Sciences and Women's Health, David Geffen School of Medicine at UCLA, Los Angeles, CA, USA, and Department of Medicine, David Geffen School of Medicine at UCLA, Los Angeles, CA, USA

E A Mayer, CNS/WH: Center for Neurovisceral Sciences and Women's Health, David Geffen School of Medicine at UCLA, Los Angeles, CA, USA, and Departments of Medicine, Physiology, and Psychiatry and Biobehavioral Sciences, David Geffen School of Medicine at UCLA, Los Angeles, CA, USA

J S Labus, J Stains, CNS/WH: Center for Neurovisceral Sciences and Women's Health, David Geffen School of Medicine at UCLA, Los Angeles, CA, USA

L Chang, CNS/WH: Center for Neurovisceral Sciences and Women's Health, David Geffen School of Medicine at UCLA, Los Angeles, CA, USA, and Department of Medicine, David Geffen School of Medicine at UCLA, Los Angeles, CA, USA

B D Naliboff, CNS/WH: Center for Neurovisceral Sciences and Women's Health, David Geffen School of Medicine at UCLA, Los Angeles, CA, USA, and Department of Psychiatry and Biobehavioral Sciences, David Geffen School of Medicine at UCLA, Los Angeles, CA, USA, and VA Greater Los Angeles Healthcare System, Los Angeles, CA, USA

Conflict of interest: None declared.

\section{REFERENCES}

1 Drossman DA, Camilleri M, Mayer EA, et al. AGA technical review on irritable bowel syndrome. Gastroenterology 2002;123:2108-31.

2 Schwetz I, Bradesi S, Mayer EA. Current insights into the pathophysiology of irritable bowel syndrome. Curr Gastroenterol Rep 2003;5:331-6.

3 Bockus HI, Bank J, Wilkinson SA. Neurogenic mucous colitis. Am J Med Sci 1928;176:813-29.

4 Hansen MB. The enteric nervous system I: organisation and classification. Pharmacol Toxicol 2003;92:105-13.

5 Elenkov IJ, Wilder RL, Chrousos GP, et al. The sympathetic nerve-an integrative interface between two supersystems: the brain and the immune system. Pharmacol Rev 2000;52:585-638.

6 Akselrod S, Gordon D, Ubel FA, et al. Power spectrum analysis of heart rate fluctuation: A quantitative probe of beat to beat cardiovascular control. Science $1981 ; 213: 220-2$.

7 Critchley HD. Electrodermal responses: What happens in the brain. Neuroscientist 2002;8:132-42.

8 Buysschaert M, Donckier J, Dive A, et al. Gastric acid and pancreatic polypeptide responses to sham feeding are impaired in diabetic subjects with autonomic neuropathy. Diabetes 1985;34:1181-5.

9 Emmanuel AV, Kamm MA. Laser Doppler flowmetry as a measure of extrinsic colonic innervation in functional bowel disease. Gut 2000;46:212-17.

10 Jarrett ME, Burr RL, Cain KC, et al. Anxiety and depression are related to autonomic nervous system function in women with irritable bowel syndrome. Dig Dis Sci 2003;48:386-94.

11 Dickhaus B, Mayer EA, Firooz N, et al. Irritable bowel syndrome patients show enhanced modulation of visceral perception by auditory stress. Am J Gastroenterol 2003;98:135-43.

12 Thompson JJ, Elsenbruch S, Harnish MJ, et al. Autonomic functioning during REM sleep differentiates IBS symptom subgroups. Am J Gastroenterol 2002;97:3147-53.

13 Gupta V, Sheffield D, Verne GN. Evidence for autonomic dysregulation in the irritable bowel syndrome. Dig Dis Sci 2002;47:1716-22.

14 Elsenbruch S, Lovallo WR, Orr WC. Psychological and physiological responses to postprandial mental stress in women with the irritable bowel syndrome. Psychosom Med 2001;63:805-10. 
15 Heitkemper M, Jarrett M, Cain KC et al. Autonomic nervous system function in women with irritable bowel syndrome. Dig Dis Sci 2001;46:1276-84.

16 Burr RL, Heitkemper $M$, Jarrett $M$, et al. Comparison of autonomic nervous system indices based on abdominal pain reports in women with irritable bowel syndrome. Biol Res Nurs 2000;2:97-106.

17 Elsenbruch S, Orr WC. Diarrhea- and constipation-predominant IBS patients differ in postprandial autonomic and cortisol responses. Am J Gastroenterol $2001 ; 96: 460-6$.

18 Orr WC, Elsenbruch S, Harnish MJ. Autonomic regulation of cardiac function during sleep in patients with irritable bowel syndrome. Am J Gastroenterol 2000;95:2865-71.

19 Adeyemi EO, Desai KD, Towsey M, et al. Characterization of autonomic dysfunction in patients with irritable bowel syndrome by means of heart rate variability studies. Am J Gastroenterol 1999:94:816-23.

20 Lee C-T, Chuang T-Y, Lu C-L, et al. Abnormal vagal cholinergic function and psychological behaviors in irritable bowel syndrome patients: A hospitalbased oriental study. Dig Dis Sci 1998;43:1794-9.

21 Waring WS, Chui M, Japp A, et al. Autonomic cardiovascular responses are impaired in women with irritable bowel syndrome. J Clin Gastroenterol 2004;38:658-63.

22 Aggarwal A, Cutts TF, Abell TL, et al. Predominant symptoms in irritable bowel syndrome correlate with specific autonomic nervous system abnormalities. Gastroenterology 1994; 106:945-50.

23 Chang L, Naliboff BD, Schmulson M, et al. The role of gender and bowel habit predominance on visceral perception in IBS. Gastroenterology 2001;120:A755

24 Naliboff BD, Berman S, Chang L, et al. Sex-related differences in IBS patients: Central processing of visceral stimuli. Gastroenterology 2003;124:1738-47.

25 Viramontes BE, Camilleri M, McKinzie S, et al. Gender-related differences in slowing colonic transit by a $5-\mathrm{HT}_{3}$ antagonist in subjects with diarrheapredominant irritable bowel syndrome. Am J Gastroenterol 2001;96:2671-9.

26 Dart AM, Du X-J, Kingwell BA. Gender, sex hormones and autonomic nervous control of the cardiovascular system. Cardiovasc Res 2002;53:678-87.

27 Bonnemeier H, Richardt G, Potratz J, et al. Circadian profile of cardiac autonomic nervous modulation in healthy subjects: Differing effects of aging and gender on heart rate variability. J Cardiovasc Electrophysiol 2003;14:791-9.

28 Chang L, Heitkemper MM. Gender differences in irritable bowel syndrome. Gastroenterology 2002;123:1686-701.

29 Mayer EA, Berman S, Chang L, et al. Sex-based differences in gastrointestinal pain. Eur J Pain 2004;8:451-63.
30 Gorman JM, Sloan RP. Heart rate variability in depressive and anxiety disorders. Am Heart J 2000;140:77-83.

31 Drossman DA, Corazziari E, Talley NJ, et al. ROME II. The functional gastrointestinal disorders, Diagnosis, pathophysiology and treatment:a multinational consensus, 2nd edn. McLean, VA: Degnon Associates, 2000

32 Zigmond AS, Snaith RP. The hospital anxiety and depression scale. Acta Psychiatr Scand 1983;67:361-70.

33 Herrmann C. International experiences with the hospital anxiety and depression scale - A review of validation data and clinical results. J Psychosom Res 1997:42:17-41.

34 Derogatis LR. SCL-90R, Administration, scoring and procedures manual-II. Towson, MD: NCS, Inc, 1983.

35 Gracely RH, McGrath P, Dubner R. Ratio scales of sensory and affective verbal pain descriptors. Pain 1978;5:5-18.

36 Munakata J, Naliboff B, Harraf F, et al. Repetitive sigmoid stimulation induces rectal hyperalgesia in patients with irritable bowel syndrome. Gastroenterology 1997; 112:55-63

37 Naliboff BD, Munakata J, Fullerton S, et al. Evidence for two distinct perceptual alterations in irritable bowel syndrome. Gut 1997:41:505-12.

38 Fallen EL, Kamath MV, Ghista DN. Power spectrum of heart rate variability: A non-invasive test of integrated neurocardiac function. Clin Invest Med 1988; 11:331-40.

39 Bagiella E, Sloan RP, Heitjan DF. Mixed-effects models in psychophysiology. Psychophysiology 2000;37:13-20.

40 Littell RC, Pendergast J, Natarajan R. Modelling covariance structure in the analysis of repeated measures data. Stat Med 2000;19:1793-819.

41 Vasey MW, Thayer JF. The continuing problem of false positives in repeated measures ANOVA in psychophysiology: A multivariate solution. Psychophysiology 1987:24:479-86.

42 Thayer JF, Siegle GJ. Neurovisceral integration in cardiac and emotional regulation. IEEE Eng Med Biol Mag 2002;21:24-9.

43 Ragnarsson G, Hallbook O, Bodemar G. Abdominal symptoms are not related to anorectal function in the irritable bowel syndrome. Scand J Gastroenterol 1999:34:250-8.

44 Westerhaus MJ, Loewy AD. Central representation of the sympathetic nervous system in the cerebral cortex. Brain Res 2001;903:117-27.

45 Benarroch EE. Central autonomic network: functional organization and clinical correlations. Armonk, NY: Futura Pub, Co., 1997.

46 Drossman DA, Morris CB, Hu Y, et al. A prospective assessment of bowel habit in irritable bowel syndrome in women: Defining an alternator. Gastroenterology 2005; 128:580-9

\title{
EDITOR'S QUIZ: GI SNAPSHOT
}

\begin{abstract}
Answer
From question on page 1376

The diagnosis was light chain deposition disease (LCDD) involving the liver.

LCDD is a rare complication of multiple myeloma occurring in only $5 \%$ of cases. Renal involvement is the most common complication of LCDD and usually dominates the clinical course. The liver is involved in at least $23 \%$ of cases but rarely affects clinical outcome. The case presented is unusual for the predominant hepatic involvement and the fact that it predated clinically significant renal disease. It is important to note however that the patient did ultimately develop acute renal failure and this more likely accounted for the noted encephalopathy than acute liver failure or portosystemic shunting. Review of the liver biopsy revealed eosinophilic material lining the sinusoids, hepatic arteries, and hepatic veins in a linear pattern. The deposits were negative for Congo red, positive for the Masson trichrome, and PAS positive diastase resistant. Immunostaining was strongly positive for $\kappa$ but negative for $\lambda$ light chains, leading to a diagnosis of $\kappa$ LCDD. LCDD is an important diagnostic consideration in all patients with myeloma who present with renal or hepatic impairment. While other causes such as drug toxicity, infection, and amyloidosis must be excluded, LCDD should be considered and the appropriate stains performed, otherwise it may be overlooked.
\end{abstract}

doi: 10.1136/gut.2004.057976 